\title{
Isolation, Characterization and Antimicrobial Activities of the Metabolites from Different Sources of Bangladesh
}

\author{
Md. Masud Rana and Md. Anwar UI Islam \\ Department of Pharmacy, University of Rajshahi, Rajshahi-6205, Bangladesh
}

Received: may 02, 2017; Accepted: May 21, 2017; Published (Web): July 31, 2017

\begin{abstract}
Antibiotic resistance and emergence of new infections are becoming serious health concern in the recent days. To overcome these problems, the present study was designed to isolate and characterize new microbial species from different locations of Rajshahi division, Bangladesh that can produce bioactive metabolites effective against resistant microorganisms. A total of 56 microbial isolates were obtained by cross streaking and agar disc diffusion method and tested against Bacillus cereus, Staphylococcus aureus ATCC-259233, Listeria monocytogenes, Agrobacterium spp., Escherichia coli FPFC-1407, Shigella dysenteriae AL-35587, Sh. sonnei, and Sh. boydii. Among the total 56 isolates, only 25\% were found to inhibit the test pathogens. One of the bacterial isolates designated as DADA-1AIMR-24 revealed maximum antimicrobial activities. From the morphological, cultural and physiological characteristics, pattern of utilization of carbon sources, growth pattern and gram staining, it was concluded that DADA-1AIMR-24 belongs to round shaped gram positive bacteria which is greyish green in color.
\end{abstract}

Key words: Isolation, Characterization, Antimicrobial Activity.

\section{Introduction}

As man faced different types of diseases from ancient times caused by infectious microorganisms so they started to search for remedies and led to the discovery of new antibiotics. Among the microorganisms, bacteria have played an important role to the health and well-being of people all over the world (Demain and Sanchez, 2009). It is the most successful source for the production of natural products that can be used in the fields of medicine, pharmacy and agriculture. In present time, most of the antibiotics produced by bacteria is used in the treatment of different infectious diseases. Different studies showed that bacteria and fungi isolated from soil are the main sources of producing new bioactive metabolites (Fenical, 1993).

But nowadays antibiotic resistance is becoming a major threat all over the world. The most resistant bacteria include methicillin resistant Staphylococcus aureus (MRSA), vancomycin resistant Staphylococcus aureus (VRSA), vancomycin resistant Enterococcus (VRE), extended spectrum lactamase producing bacteria such as E. coli and Klebsiella spp. and multiple drug resistant Mycobacterium tuberculosis (Sharma et al., 2011a). In $21^{\text {st }}$ century drug resistance has become a major healthcare problem (Alanis, 2005). Bacteria acquire resistance by destroying drugs, developing drug tolerance, mutation, conjugation, transduction, transformation and transposition (Raghunat, 2008; Maragakis et al., 2008). By the study of 2001, it has shown that more than $70 \%$ of pathogenic bacteria were estimated to be resistant to at least one of the currently available antibiotics (Cragg and Newman, 2001). The prevalence of drug resistance leads to substantial morbidity and mortality among the infant, elderly and immuno-suppressive patients (Barsby et al., 2001; Parungao et al., 2007). The consequence of

Correspondence to: Md. Anwar Ul Islam; E-mail: profanwarulislam@yahoo.com;Tel: +88-0721-750071; 
drug resistance affect the duration of treatment, threaten health security, damage trade and economics (Singer et al., 2003). Due to drastically increasing of drug resistance it is urgently needed to discover new antimicrobial agents that act against multidrug resistance and different types of infections (Gusky and Tsuji, 2010).

Therefore, it is essential to search for new, efficacious and safe antibiotics from natural sources to combat the risk of drug resistant infections. So, the objective of the present study was to screen soil samples collected from different areas of Rajshahi division which are large, diverse and largely unscreened ecosystems, for the isolation of potent and broad-spectrum antibiotic producing bacterial strain (Sharma et al., 2011b).

However, our main aim in this study was to investigate indigenous soil resources having potent antimicrobial compound producing ability that could be used to produce new product with better efficacies (Hassan et al., 2014).

\section{Materials and Methods}

Sampling sites and collection of soil samples: Soil samples were collected from the different location of Rajshahi division, Bangladesh for screening of microorganisms with inhibitory activity against other pathogenic organisms. Samples were collected as aseptically as possible. Samples were collected from various depth of the earth surface, ranging from layers just beneath the upper surface to 1.5 feet depth. A trowel was used to dig the soil. The samples were collected in the sterile small plastic tubes and properly labeled indicating the date of collection and the depth. Four soil samples were collected in seven days (08-14 June, 2015). The collected soil samples were dried in a hot air oven at $60-65^{\circ} \mathrm{C}$ for about three hours and stored at $4^{\circ} \mathrm{C}$ for further research work.

Isolation of pure culture of isolates: To isolate pure culture of isolates, we used standard microbiological method (spread plate technique). Fifty six microbial strains were isolated and obtained as pure culture. For the isolation of pure microbial strain, serial dilution techniques were used by tenfold dilution methods (Nonomura et al., 1969). In this method, $1 \mathrm{~g}$ of dried soil was suspended in $9 \mathrm{ml}$ sterile water and consecutive serial dilutions were made by transferring $1 \mathrm{ml}$ of aliquots to $2^{\text {nd }}$ test tube containing $9 \mathrm{ml}$ of sterile water and in this way dilution was carried out up to $10^{4}$ times. Then using vortex, uniform suspensions of the contents were made. From each dilution $0.1 \mathrm{ml}$ aliquot was taken carefully and spread uniformly over the surface of isolation medium containing nystatin $(100 \mu \mathrm{g} / \mathrm{ml})$ on $16 \mathrm{~cm}$ petri dishes. In this study starch-casein nitrate agar medium were used as isolation medium. After completing the process the plates were wrapped by parafilm and incubated at $37^{\circ} \mathrm{C}$ and monitored for 7 days. Finally the colonies were carefully counted by visual observation.

Preservation of the pure isolates: After isolating, the pure isolates were preserved. For short time preservation yeast-extract glucose agar slants were used. Then the microbial strains were inoculated in yeast-extract glucose agar slants using a sterile loop and incubated at $37^{\circ} \mathrm{C}$ for 5 days. After that the yeastextract glucose agar slants containing purified organisms were kept in a refrigerator at $4^{0} \mathrm{C}$ for short time preservation (two months). But for long term preservation the pure isolates were mixed by vortex mixer and kept at $-20^{\circ} \mathrm{C}$ in the presence of glycerol $(15 \% \mathrm{v} / \mathrm{v})$.

Screening of pure isolates for antibacterial activity (cross streaking and plug technique): The pure isolates were preliminary screened for antibacterial activity by using cross streaking and plug technique on yeast-extract glucose agar medium (Alcamo et al., 2004). Each pure isolate was streaked individually in straight line on different agar plates. Then the plates were incubated at $37^{0} \mathrm{C}$ for 5 days to allow the isolates to secrete sufficient antibacterial metabolites into the medium. After the incubation period, diluted test organisms were streaked perpendicularly on the same plate of the isolates. After that the plates were incubated at $37^{\circ} \mathrm{C}$ for 24 hours. Finally the plates were examined and the zone 
of inhibition around the isolates was measured using a millimeter scale (Shomura et al., 1980).

Test organisms: In this study we used four grampositive and four gram-negative bacteria. Bacillus cereus, Staphylococcus aureus ATCC-259233, Listeria monocytogenes and Agrobacterium sp. were the gram-positive bacteria and Escherichia coli FPFC-1407, Shigella dysenteriae AL-35587, Shigella sonnei and Shigella boydii were the gram negative bacteria. The test fungi were Aspergillus niger, Tichiderma viridae and Microphomina phaseolina. The test bacteria were previously collected from International Centre for Diarrhoeal Disease Research, Bangladesh by Pharmacy Department at Rajshahi University and the test fungi were collected from Botany Department at Rajshahi University.

Characterization of potential strain: The isolated strain designated as DADA-1AIMR-24 showing potent antimicrobial activities were subjected for morphological, cultural and physiological characteristics to identify the strain. The morphological, cultural and physiological characteristics of the strains were determined in accordance with the method described by Shirling and Gottlieb, 1966. Microscopic characterization was carried out by cover slip culture method (William and Davis, 1967). Cultural characteristics were tested in tryptone-yeast extract agar (ISP-1), yeast extract-malt extract agar (ISP-2), inorganic salt-starch agar (ISP4), glycerol-asparagine agar (ISP-5), tyrosine agar (ISP-7), nutrient agar, yeast extract-glucose agar, czapek-dox agar (acidic) and czapek-dox agar (basic). The physiological characteristics were examined in yeast-extract glucose agar media for melanoid production, liquefaction of gelatin, hydrolysis of starch, decomposition of cellulose, nitrate reduction, and $\mathrm{NaCl}$ tolerance. The utilization of carbon sources was also tested on ISP-9 medium.

Gram staining of bacterial strain DADA-1AIMR-24: Gram staining of DADA-1AIMR-24 was performed according to the standard procedure.

\section{Results and Discussions}

Total 56 microbial strains were isolated from soil samples of Rajshahi division, Bangladesh. Microbial colonies in isolation plate are shown in figure 1. These were designed as AIMR-1 to AIMR-9, AIMR50 to AIMR-55, AIMR-13 to AIMR-44, AIMR-60 to AIMR-67 and DADA-1AIMR-24 (Table 1).

Table 1. Collection site, depth and number of microbial colonies per gram of soil.

\begin{tabular}{llll}
\hline Date of Collection & Collection site & Depth of the soil & Isolates \\
\hline 08/06/2015 & Vegetable garden, TMSS, Bogra. & 6 inch & AIMR-1 to AIMR-9 \\
09/06/2015 & Farmland, TMSS, Bogra. & 4 inch & AIMR-50 to AIMR-55 \\
$10 / 06 / 2015$ & Medicinal plant garden, RU campus & 1 feet & AIMR-13 to AIMR-44 \\
$14 / 06 / 2015$ & Bank of pond, TMSS, Bogra. & 8 inch & AIMR-60 to AIMR-67 \\
$16 / 06 / 2015$ & Collected from Supervisor's reserve & isolated previously in & DADA-1AIMR-24 \\
& sample (DADA-1) & our lab. & \\
\hline
\end{tabular}

Among the 56 isolates, 14 were found to have antibacterial activities against a wide range of Grampositive and Gram-negative bacteria. This indicated that these 14 strains produce antimicrobial compounds in the culture media when incubated for a defined period of time. Among the following isolates, DADA-1AIMR-24 were selected due to inhibition of growth of the test pathogenic organisms with significant potency. The potential strain DADA-
1AIMR-24 was identified by morphological, cultural, physiological and utilization of carbon source study. The complete data was reported in tables 2, 3 and 4 .

The antifungal activities of bacterial strain DADA-1AIMR-24 also found against Aspergillus niger, Tichiderma viridae, Microphomina phaseolina.

DADA-1AIMR-24 grew very slowly at first day and then at $2^{\text {nd }}$ day it grew moderately and green 
colored appeared and gradually turned into thick. As the time passed, the isolate became more greyish green due to pigmentation of the bacterial strain. In

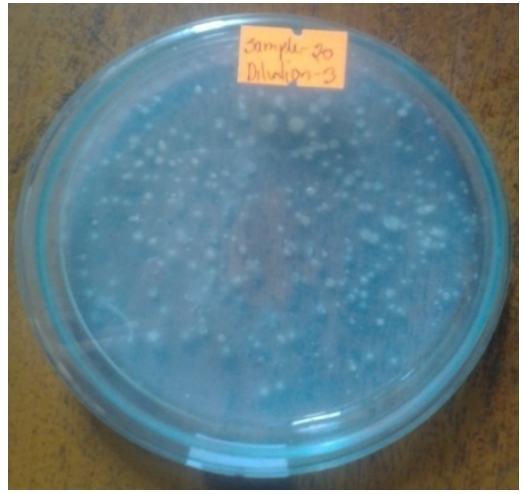

Dilution 3 cultural characteristics, the strain grew rapidly and abundantly in YEGA medium.

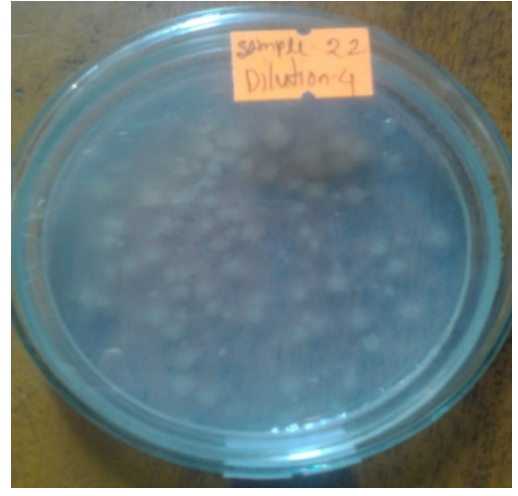

Dilution 4

Figure 1. Colonies of microorganisms appeared on the dilution plates of the soil samples.

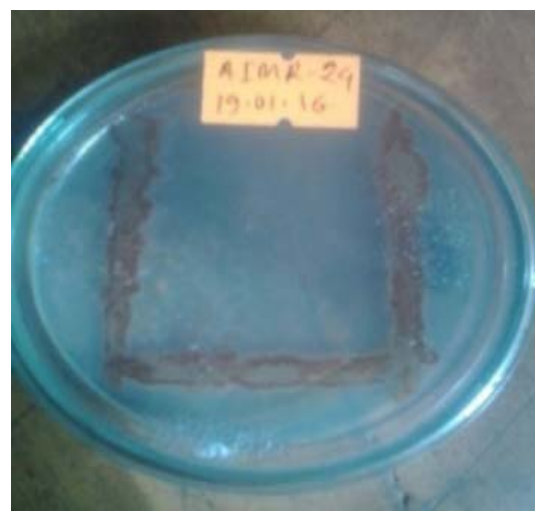

DADA-1AIMR-24

Figure 2. Bacterial Isolate from samples DADA-1.

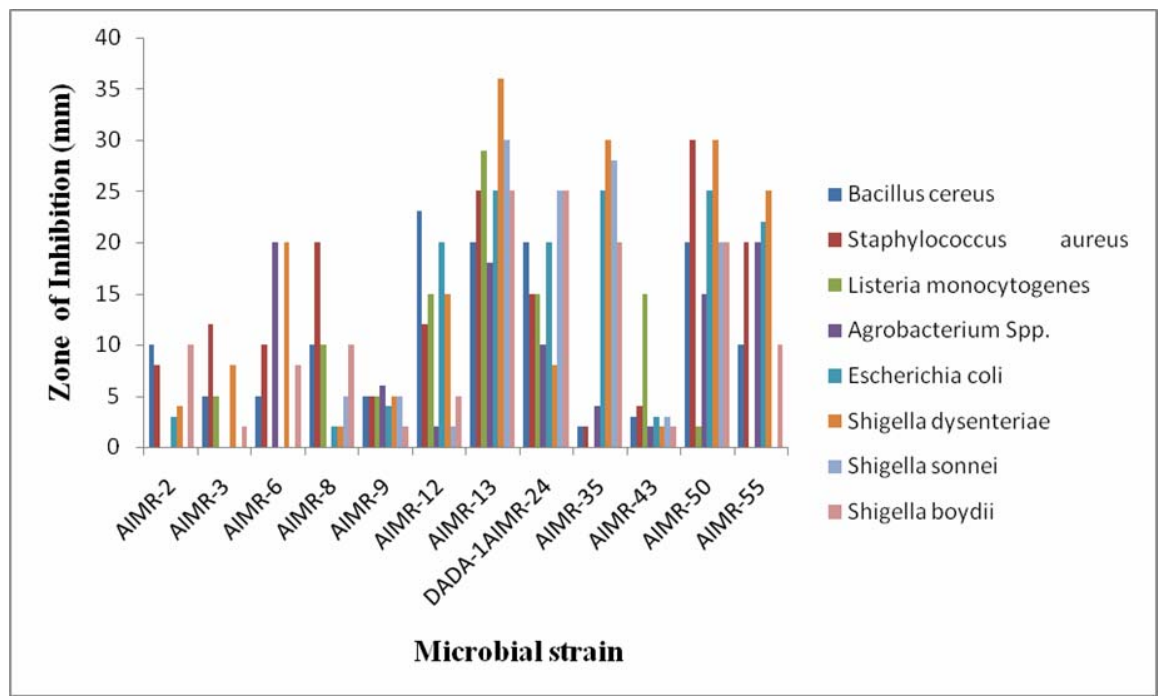

Figure 3. Antibacterial activities of isolates (only active isolates have been shown). 
The antibacterial activity of DADA-1AIMR-24 found against Bacillus cereus (Plug technique).

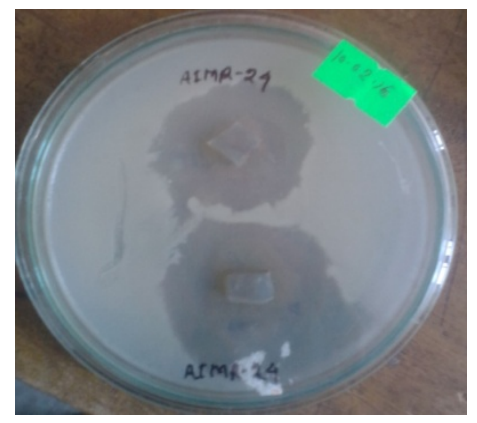

DADA-1AIMR-24

Figure 4. Screening for the antibacterial activity of the isolate through plug technique against Bacillus cereus in yeast-extract glucose agar medium.

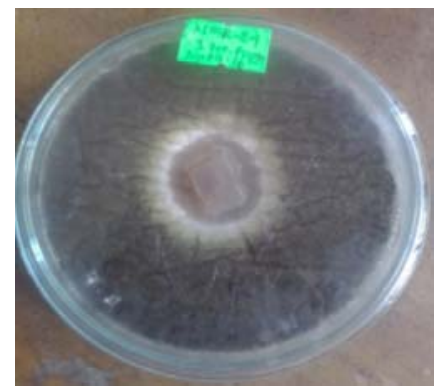

A

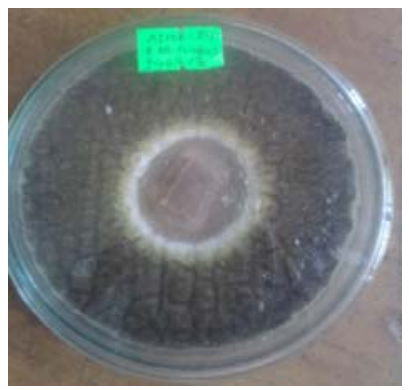

B

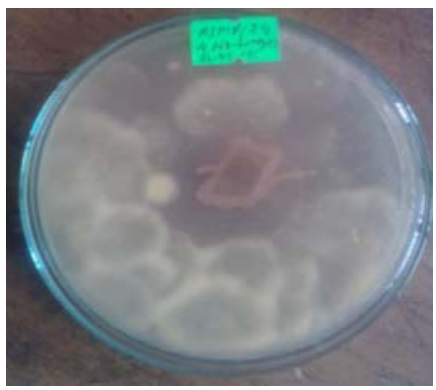

C

Figure 5. Screening for the antifungal activities of the isolate through plug technique against (A) Aspergillus niger, (B) Tichiderma viridae and (C) Microphomina phaseolina in potato dextrose agar medium.

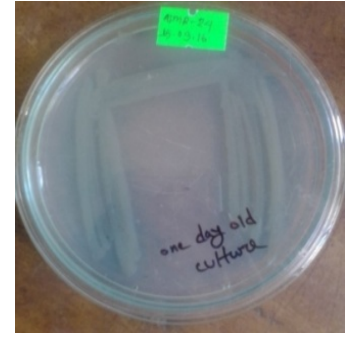

One day old culture

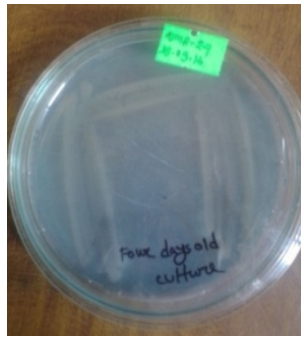

five days old culture

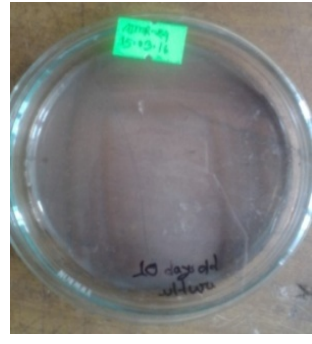

seven days old culture

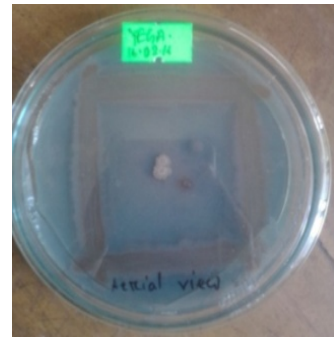

Ten days old culture

Figure 6. Aerial mycelial view of DADA-1AIMR-24 (on $1^{\text {st }}, 5^{\text {th }}, 7^{\text {th }}, 10^{\text {th }}$ days) on yeast-extract glucose agar medium after incubation.

The bacterial strain DADA-1AIMR-24 showed round shaped microscopic view.

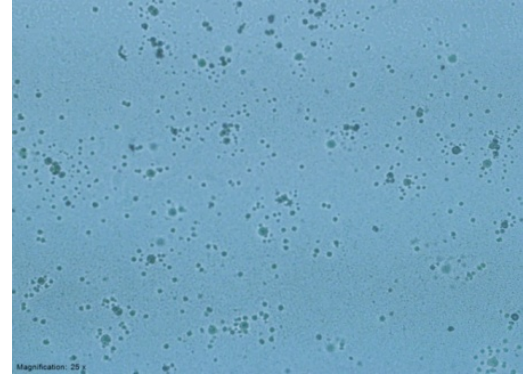

Four days old culture (200)

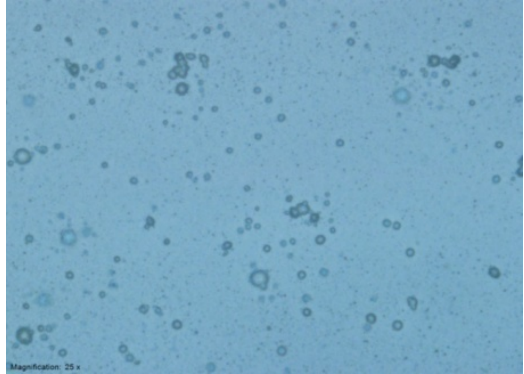

Four days old culture (400)

Figure 7. Microscopic view of bacterial strain DADA-1AIMR-24 at magnification $\times 200$ and at magnification $\times 400$ on yeast-extract glucose agar media. 
The bacterial strain DADA-1AIMR-24 retained crystal violet color which indicated that the strain DADA-1AIMR-24 is gram-positive.

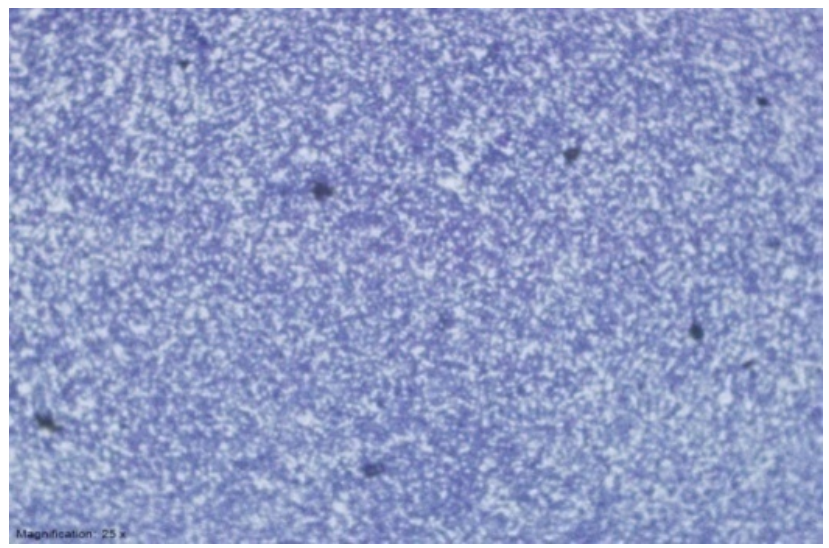

Figure 8. Gram staining of DADA-1AIMR-24

Table 2. Cultural characteristics of strain DADA-1AIMR-24 (Incubation period: 7 days, Temp: $37^{0} \mathrm{C}$ ).

\begin{tabular}{|c|c|c|c|c|}
\hline Medium & Growth & Aerial mycelium & $\begin{array}{l}\text { Reverse side substrate } \\
\text { mycelium }\end{array}$ & $\begin{array}{c}\text { Diffusible } \\
\text { pigment }\end{array}$ \\
\hline Tryptone-yeast extract agar (ISP-1) & Moderate & Dark reddish brown & Dusky blue green & + \\
\hline Yeast extract-malt extract agar (ISP-2) & Abundant & Dusky red & Dusky red & ++ \\
\hline Inorganic salt-starch agar (ISP-4) & Low & White & White & - \\
\hline Glycerol-asparagine agar (ISP-5) & Low & Dusky yellow & White & + \\
\hline Tyrosine agar (ISP-7) & Moderate & Grayish black & Brownish black & ++ \\
\hline Yeast-extract glucose agar (YEGA) & Abundant & Light green & Blackish red & +++ \\
\hline Nutrient agar & Moderate & Dark greenish gray & Dark yellowish green & + \\
\hline Czapek-dox agar acidic (pH 5.3) & Low & White & White & - \\
\hline Czapek-dox agar basic (pH 8) & Low & White & White & - \\
\hline
\end{tabular}

Legend: +++=High pigment, ++=Moderate pigment and += Low pigment, - = no pigment

Table 3. physiological characteristics of the strain DADA-1AIMR-24.

\begin{tabular}{lc}
\hline Properties & Results \\
\hline Temperature range for growth & $25-45^{\circ} \mathrm{C}$ \\
Optimum temperature for growth & $30-40^{\circ} \mathrm{C}$ \\
Liquefaction of gelatin & + \\
Hydrolysis of starch & ++ \\
Melanoid production & + \\
Decomposition of cellulose & + \\
Nitrate reduction & + \\
\multicolumn{2}{c}{ NaCl tolerance } \\
Keratolytic activity & $0-2 \%$ \\
\hline
\end{tabular}

Legend: ++= positive, += weakly positive and -= negative. 
Table 4. Utilization of carbon sources by the strain DADA-1AIMR-24.

\begin{tabular}{lcc}
\hline Carbon source & Utilization & production of pigments \\
\hline D-glucose & + & ++ \\
D-fructose & + & +++ \\
D-xylose & + & + \\
Lactose & + & +++ \\
Sucrose & + & + \\
Mannitol & + & +++ \\
Inositol & + & +++ \\
L-Rhamnose & + & +++ \\
No addition & - & - \\
\hline
\end{tabular}

Note, $+++=$ abundant production, $++=$ moderate production, $+=$ low production .

\section{Conclusion}

The ultimate goal of this study was to search antimicrobial metabolites producing new microbial strain. In our study, 56 microbial strains were isolated. The initial screening by both cross streaking and plug technique indicates that the 16 isolates give antibacterial activity. Among them DADA-1AIMR24, AIMR-66 and AIMR-67 produce a very potent antibacterial metabolites that inhibited the growth of wide range of test bacteria and only DADA-1AIMR24 shows antifungal activity against pathogenic fungi.

Due to strong antibacterial and antifungal activity of DADA-1AIMR-24, we selected it for further study. In order to identify this active bacterial strain further study will be needed. But16S rDNA sequence is also needed to confirm its species level. However, from above discussion we can say that Bangladesh is rich in antimicrobial metabolites producing bacteria and extensive research in this field may discover novel bacterial species capable of producing novel bioactive compounds.

\section{Declaration of Interests}

The authors declare that they have no conflicts of interests.

\section{Acknowledgements}

We are grateful to the ministry of National Science and Technology (NST), Government of
Bangladesh, for funding us to carry out this work. Finally we are deeply thankful to Ms Sherin Sultana, Lecturer in Khademul Islam Girls' College, Rajshahi for allowing us to work on DADA-1 sample.

\section{References}

Alanis, AJ. 2005. Resistance to antibiotics: are we in the post-antibiotic era? Arch Med. Res. 36, 697-705.

Alcamo, E. and Pomerville, J.C. 2004. Alcamo's laboratory fundamental of microbiology. $7^{\text {th }}$ Edn, Jones \& Bartlett Publishers, London

Barsby, T., Kelly, M.T., Gagne, S.M. and Andersen, R.J. 2001. Bogorol a produced in culture by a marine Bacillus sp. reveals a novel template for cationic peptide antibiotics. Org. Lett. 3, 437-440.

Cragg, G.M. and Newman, D.J. 2001. Medicinals for the millennia: The historical record. Ann. N. Y. Acad. Sci. 953, 3-25.

Demain, AL and Sanchez S. 2009. Microbial drug discovery: 80 years of progress. J. Antibio. 62, 5-16.

Fenical, W. 1993. Chemical studies of marine bacteria: developing a new resource. Chem. Rev. 93, 16731683.

Gusky, M.T. and Tsuji, B.T. 2010. A comparative review of the lipoglycopeptides: oritavancin, dalbavancin and telavancin. Pharmacotherapy. 30, 80-94.

Hassan, S.A., Hanif, E. and Zohra, R.R. 2014. Isolation and Screening of soil bacteria for potential antimicrobial activity. Fuuast J. Biol. 4, 217-219.

Maragakis, L.L., Perencevich, E.N. and S.E. Cosgrove, .S.E. 2008. Clinical and economic burden of antimicrobial resistance. Expert Rev. Anti Infect. Ther. 6, 751-763. 
Nonomura and Ohara 1969. Developing a new resource for drug discovery. Mar. actin. 9, 79-105

Parungao, M.M., Maceda, E.B.G. and Villano, M.A.F. 2007. Screening of antibiotic producing actinomycetes from marine, brackish and terrestrial sediment of Samal Island, Philippines. J. Res. Sci. Comput. Eng. 4, 29-38.

Raghunath, D. 2008. Emerging antibiotic resistance in bacteria with special reference to India. J. Biosci. 33, 593-603.

Sharma, D., Kaur T., Chadha, BS. and Manhas, R. K. (2011a,b). Antimicrobial Activity of Actinomycetes Against Multidrug Resistant Staphylococcus aureus, E. coli and Various Other Pathogens. Trop. J. Pharm. Res. 10, 801-808.

Shirling, E.B. and Gottlieb, D. 1966. Methods of characterization of Streptomyces species Inter. J. Systemic Bacteriol. 16, 313-340.
Shomura, T., Omoto, S.M., Ohba, K. and Ogino, H. 1980. "SF-1961, a new antibiotic related to bleomycin. $J$. Antibio. 11, 1243-1248.

Singer, R.S., Finch, R., Wegener, H.C., Bywater, R., Walters, J. and Lipsitch, M. 2003. Antibiotic resistance-the interplay between antibiotic use in animals and human beings. T. Lan. Infect. Dis. 3, 47-51.

Tawiah, A.A., Gbedema, S.Y., Adu, F., Boamah, V.E. and Annan, K. 2012. Antibiotic producing microorganisms from River Wiwi, Lake Bosomtwe and the Gulf of Guinea at Doakor Sea Beach, Ghana. BMC Microbiology. 12, 234-241.

Williams, S.T. and Davies, F.L. 1967. Use of a scanning electron microscope for the examination of actinomycetes, J. Gen. Microbiol. 48, 171-177. 